\title{
Breaking of Periodicity at Positive Temperatures
}

\author{
A. C. D. van Enter ${ }^{1}$ and J. Miẹkisz ${ }^{2, \star}$ \\ ${ }^{1}$ Institute for Theoretical Physics, P.O. Box 800, University of Groningen, Groningen, \\ The Netherlands \\ 2 Department of Mathematics, University of Missouri, Columbia, Missouri 65211, USA
}

Received April 16, 1990

\begin{abstract}
We discuss a classical lattice gas model without periodic or quasiperiodic ground states. The only ground state configurations of our model are nonperiodic Thue-Morse sequences. We show that low temperature phases of such models can be ordered. In fact, we prove the existence of an ordered (nonmixing) low temperature translation invariant equilibrium state which has nonperiodic Gibbs states in its extremal decomposition.
\end{abstract}

A number of classical lattice gas models without periodic ground state configurations have been found recently. So far, they were of quasiperiodic (in the sense described below) or incommensurate character. In the first category we have twodimensional nearest neighbor interactions studied recently by Radin and Miękisz [1-9] (for related ideas in a somewhat different setup see $[10,11])$. The models are based on results on nonperiodic tilings of the plane with a finite family of squarelike tiles [12-15]. The ground state configurations of these models are nonperiodic structures minimizing the energy of all bonds simultaneously (interactions are not competing). They are quasiperiodic in the following sense: if a certain fraction of particles is ignored the rest of a ground state configuration is periodic; the smaller the fraction, the larger the period. Incommensurate ground state configurations, on the other hand, arise as a result of competition between interactions with incommensurate length scales $[16,17]$.

Recently, an example was given of a one-dimensional exponentially decaying Ising-type non-competing interaction with ground state configurations which are neither quasiperiodic nor incommensurate [18]. The unique ground state (the unique translation invariant probability measure supported by ground state configurations) of this model has two unusual properties: it has perfect fractal symmetry in the sense that the structure of the ground state is invariant under certain scale changes and the translation (shift) operator has some continuous spectrum. The ground state, as in the previous models, is not mixing. We say that it has long range order in the sense that distant regions are correlated.

\footnotetext{
* Present address: Institut de Physique Théorique, Université de Louvain-la-Neuve, Belgium
} 
The problem we will be addressing here is that of the low temperature stability of the nonperiodic ground state of this model. We will show that there is an (in some sense similar) interaction with the same unique ground state and an ordered (nonmixing) low temperature translation invariant equilibrium state which has nonperiodic Gibbs states in its extremal decomposition.

Let us now describe this one-dimensional model in detail [18]. The spin variable at site $j$ of $\mathbf{Z}$ will be denoted by $\sigma_{j}$, and can attain the values \pm 1 . The Hamiltonian is a translation invariant 4 -spin interaction of the form

$$
\begin{aligned}
H= & \sum_{j=-\infty}^{\infty} \sum_{p=0}^{\infty} \sum_{r=0}^{\infty} V(j, p, r)=\sum_{j=-\infty}^{\infty} \sum_{p=0}^{\infty} \sum_{r=0}^{\infty} \\
& \times \exp -(r+p)^{2}\left[\sigma_{j}+\sigma_{j+2^{r}}\right]^{2}\left[\sigma_{j+(2 p+1) 2^{r}}+\sigma_{j+(2 p+2) 2^{r}}\right]^{2} .
\end{aligned}
$$

Ground state configurations of this Hamiltonian are Thue-Morse sequences [19]. They can be obtained from the configuration equal to +1 everywhere by the following recursive procedure. At the first step we flip every second spin. At the $n^{\text {th }}$ step we flip all blocks of $2^{n}$ spins within the previous $n-1^{\text {st }}$ configuration from site $(2 k+1) 2^{n}+1$ to $(2 k+2) 2^{n}$ for every $k$. The limit of this sequence of periodic configurations of period $2 \cdot 2^{n}$ is a nonperiodic sequence. The closure of its orbit under translation supports only one translation invariant measure which is strictly ergodic [19]. We will call this measure $\mu_{M}$. This is the unique ground state of the above Hamiltonian [18]. The ground state energy density is equal to zero. All this Hamiltonian does is to assign positive energy to certain 4-point patterns. ThueMorse sequences are the only sequences which do not contain any of these patterns. It is easy to see that $\mu_{M}$ is not mixing. Let $P_{j}$ and $Q_{j}$ be projections on configurations with spin + or - respectively at the site $j: P_{j}=\frac{1}{2}\left(\sigma_{j}+1\right)$, $Q_{j}=\frac{1}{2}\left(1-\sigma_{j}\right)$. It was proved in [18] that

$$
\begin{aligned}
& \mu_{M}\left(P_{0} P_{2^{r}} P_{(2 p+1) 2} P_{(2 p+2) 2}\right)=0, \\
& \mu_{M}\left(Q_{0} Q_{2^{r}} Q_{(2 p+1) 2} Q_{(2 p+2) 2^{r}}\right)=0, \\
& \mu_{M}\left(P_{0} P_{2^{r}} Q_{(2 p+1) 2^{r}} Q_{(2 p+2) 2^{r}}\right)=0, \\
& \mu_{M}\left(Q_{0} Q_{2^{r}} P_{(2 p+1) 2^{r}} P_{(2 p+2) 2^{r}}\right)=0,
\end{aligned}
$$

and $\mu_{M}\left(P_{0} P_{1}\right)>0$, therefore choosing $r=0$

$$
\mu_{M}\left(P_{0} P_{1} P_{2 p+1} P_{2 p+2}\right) \nrightarrow\left(\mu_{M}\left(P_{0} P_{1}\right)\right)^{2}
$$

as $p \rightarrow \infty$ so $\mu_{M}$ is not mixing.

We will prove that for every $\varepsilon>0$ there is a low temperature translation invariant equilibrium state $\varrho_{U}$ for some interaction $U$ such that

$$
\begin{aligned}
& \varrho_{U}\left(P_{0} P_{2^{r}} P_{(2 p+1) 2^{r}} P_{(2 p+2) 2^{r}}\right) \leqq \varepsilon, \\
& \varrho_{U}\left(Q_{0} Q_{2^{r}} Q_{(2 p+1) 2^{r}} Q_{(2 p+2) 2^{r}}\right) \leqq \varepsilon, \\
& \varrho_{U}\left(P_{0} P_{2^{r}} Q_{(2 p+1) 2^{r}} Q_{(2 p+2) 2^{r}}\right) \leqq \varepsilon, \\
& \varrho_{U}\left(Q_{0} Q_{2^{r}} P_{(2 p+1) 2^{r}} P_{(2 p+2) 2^{r}}\right) \leqq \varepsilon
\end{aligned}
$$

uniformly in $p$ and $r$. It follows that $\varrho_{U}$ is not mixing. Otherwise, taking $p \rightarrow \infty$ and $r=0$ we would obtain from $(7)$ and $(8)$ that $\varrho_{U}\left(P_{0} P_{1}\right) \leqq \sqrt{\varepsilon}$ and $\varrho_{U}\left(Q_{0} Q_{1}\right) \leqq \sqrt{\varepsilon}$. Thus the density of nearest neighbor pairs of spins pointing in the same direction could be made arbitrarily small. The same argument, with $r=1$, would cause also the density of next nearest neighbor pairs of spins pointing in the same direction to 
become arbitrarily small. It is obvious that these two constraints cannot be satisfied at the same time, hence $\varrho_{U}$ cannot be mixing.

To show (7-10) we will use the method introduced by Israel [20-22] and based on the Bishop-Phelps theorem about approximations of translation invariant states by translation invariant equilibrium states [23]. Let us state the relevant corollary of this theorem [21].

Proposition 1. Let $U_{0}$ be any summable interaction (the sum of absolute values of all coupling constants is finite), $\varrho_{0}$ any translation invariant state (probability measure) on the space of configurations, $\mathscr{F}$ the closed convex cone generated by some interactions $V_{i}$ (the closure of the set of linear combinations of $V_{i}$ with positive coefficients) and finally $\varepsilon>0$. Then there is a translation invariant equilibrium state $\varrho_{U}$ for some interaction $U \in U_{0}+\mathscr{F}$ such that $\varrho_{U}(W) \leqq \varrho_{0}(W)+\varepsilon\|W\|$ for all $W$ in the cone $\mathscr{F}$.

Inequalities (7-10) follow when we identify $U_{0}$ with $H, \varrho_{0}$ with $\mu_{M}$ and $V_{i}$ with the observables in (7-10). The perturbed summable 4-spin interaction assigns a positive energy to exactly the same 4-point patterns as $H$ does, so $\mu_{M}$ is again its only ground state and $\varrho_{U}$ its ordered (nonmixing) low temperature translation invariant equilibrium state. We have proved the following theorem concerning the low temperature stability of the Thue-Morse state.

Theorem 1. There is a 4-spin summable interaction $U$ with the Thue-Morse state as its only ground state and with a low temperature translation invariant nonmixing equilibrium state $\varrho_{U}$.

Now we will show that $\varrho_{U}$ has nonperiodic Gibbs states in its extremal decomposition. First, let us observe that for every translation invariant probability measure and in particular for $\varrho_{U}$ the density of pairs of spins at a distance $2^{r}$ pointing in the same direction and the density of pairs of spins at a distance $2^{r+1}$ pointing in the same direction cannot be both arbitrarily small for any $r$. More formally, let $S_{j}^{r}=P_{j} P_{j+2^{r}}+Q_{j} Q_{j+2^{r}}$. It is easy to see that either

or

$$
\varrho_{U}\left(S_{j}^{r}\right) \geqq \frac{1}{8}
$$

$$
\varrho_{U}\left(S_{j}^{r+1}\right) \geqq \frac{1}{8}
$$

Theorem 2. In the decomposition into extremal Gibbs states of $\varrho_{U}$ there are nonperiodic Gibbs states.

Proof. Let $\varrho_{U}=\int \varrho \mu(d p)$ be the decomposition of $\varrho_{U}$ into extremal Gibbs states; $\varrho_{U}$ is a barycenter of $\mu$, a measure supported by extremal Gibbs states of the interaction $U$. Let $B_{j}$ be the set of all extremal Gibbs states which are not periodic with period smaller or equal to $j$. Obviously, $B_{j+1} \subset B_{j}$ and $B=\bigcap_{j=1}^{\infty} B_{j}$ is the set of all nonperiodic extremal Gibbs states. We will show that $\mu(B)>0$. Fix $\varepsilon>0$ and assume on the contrary that $\mu(B)=0$, hence $\mu\left(B_{j}\right) \rightarrow 0$ as $j \rightarrow \infty$, so there exists $j_{0}$ such that $\mu\left(B_{j_{0}}\right)<\varepsilon$. Choose $r$ such that (11) holds and $j_{0} \leqq 2^{r}$ and $p$ such that $2^{r}(2 p+1)$ !! contains all numbers up to $j_{0}$ as its divisors. Let us recall that if $\varrho$ is an extremal periodic Gibbs state with a period $j$ then $\varrho\left(S_{0}^{r} S_{n j}^{r}\right) \rightarrow\left(\varrho\left(S_{0}^{r}\right)\right)^{2}$ for every $r$ when $n \rightarrow \infty$. Let

$$
A_{n}^{j}=\left\{\varrho: \varrho \text { has period } j, \varrho\left(S_{0}^{r} S_{k j}^{r}\right)<\varrho\left(S_{0}^{r}\right)^{2}-\varepsilon \text { for some } k \geqq n\right\} \text {. }
$$


Obviously, $A_{m}^{j} \subset A_{n}^{j}$ if $m>n . A^{j}=\bigcap_{n=1}^{\infty} A_{n}^{j}$ does not contain any extremal Gibbs states so $\mu\left(A^{j}\right)=0$ and therefore $\mu\left(A_{n}^{j}\right) \rightarrow 0$ as $n \rightarrow \infty$ for all $j$. Choose $n(j)$ such that $\mu\left(A_{n(j)}^{j}\right)$ $<\varepsilon(j)$ and $\sum_{j=1}^{j_{0}} \varepsilon(j)<\varepsilon$. Now, let $n>\max (n(j))$ and let $n$ be an odd number, then $\varrho_{U}\left(S_{0}^{r} S_{2^{r(2 p+1) ! ! n}}^{r}\right)>\frac{1}{64}-3 \varepsilon$ which contradicts $(7-10)$ for small $\varepsilon$.

We can also prove the existence of nonperiodic Gibbs states for 2-spin summable interactions. In order to do so we modify slightly the above example. We will approximate not $\mu_{M}$ but a generalized Thue-Morse state $\mu_{K}$ [19]. It can be constructed as before with the following change that at the $n^{\text {th }}$ step we flip all blocks of $n$ ! spins from site $(2 k+1) n !+1$ to $(2 k+2) n$ ! for every $k$. We know a 2 -spin summable interaction without periodic ground state configurations for which $\mu_{K}$ is a ground state. We cannot prove, however, that it is its only ground state. The Hamiltonian consists of antiferromagnetic interactions at distances $n$ ! for every $n$. Such interactions were used to prove that generically, in the space of summable interactions, ground state configurations are ordered (nonmixing) but not periodic [24-26]. For related ideas see also [27-32]. It follows from Proposition 1 that for every $\varepsilon>0$ there is a 2 -spin summable antiferromagnetic interaction $V$ with a translation invariant equilibrium state $\varrho_{V}$ such that

$$
\limsup _{n \rightarrow \infty} \varrho_{V}\left(\sigma_{0} \sigma_{n !}\right)<-1+\varepsilon .
$$

Using only 2-point correlation functions and repeating the proof of Theorem 2 one can show that there are nonperiodic Gibbs states in the extremal decomposition of $\varrho_{V}$.

In summary we note that we have shown that a ground state which is neither periodic nor quasiperiodic gives rise to an ordered low temperature translation invariant equilibrium state which has nonperiodic Gibbs states in its extremal decomposition. These nonperiodic extremal Gibbs states build an extremal translation invariant equilibrium state. They are therefore qualitatively different from Dobrushin's extremal nonperiodic states describing the coexistence of phases in the three-dimensional ferromagnetic Ising model [33]. It remains an open question whether a translation invariant interaction without periodic ground state configurations and without periodic extremal Gibbs states exists.

Acknowledgements. The research of A.C.D. van Enter has been made possible by a fellowship of the Royal Netherlands Academy of Arts and Sciences. We thank Charles Radin for discussions.

\section{References}

1. Radin, C.: Tiling, periodicity, and crystals. J. Math. Phys. 26, 1342-1344 (1985)

2. Radin, C.: Crystals and quasicrystals: a lattice gas model. Phys. Lett. 114 A, 381-383 (1986)

3. Radin, C.: Crystals and quasicrystals: a continuum model. Commun. Math. Phys. 105, 385-390 (1986)

4. Miękisz, J., Radin, C.: The unstable chemical structure of the quasicrystalline alloys. Phys. Lett. 119 A, 133-134 (1986)

5. Miẹkisz, J.: Many phases in systems without periodic ground states. Commun. Math. Phys. 107, 577-586 (1986)

6. Miękisz, J.: Classical lattice gas model with a unique nondegenerate but unstable periodic ground state configuration. Commun. Math. Phys. 111, 533-538 (1987)

7. Radin, C.: Low temperature and the origin of crystalline symmetry. Int. J. Mod. Phys. B 1, 1157-1191 (1987) 
8. Miẹkisz, J.: Toward a microscopic model of a quasicrystal. Phys. Lett. 138 A, 415-416 (1989)

9. Miękisz, J.: A microscopic model with quasicrystalline properties. To appear in J. Stat. Phys. (1990)

10. Ruelle, D.: Thermodynamic Formalism, Sect. 4.15. New York: Addison-Wesley 1978

11. Schlijper, A.G.: Tiling problems and undecidability in the cluster variation method. J. Stat. Phys. 50, 689-714 (1988)

12. Berger, R.: The undecidability of the domino problem. Mem. Am. Math. Soc. 66; (1966)

13. Robinson, R.M.: Undecidability and nonperiodicity for tilings of the plane. Invent. Math. 12 177-209 (1971)

14. Myers, D.: Nonrecursive tiling of the plane II. J. Symb. Log. 39, 286-294 (1974)

15. Grunbaum, B., Shephard, G.C.: Tilings and patterns. New York: Freeman 1986

16. Bak, P., Bruinsma, R.: One-dimensional Ising model and the complete Devil's staircase. Phys. Rev. Lett. 49, 249-251 (1982)

17. Aubry, S.: Exact models with a complete Devil's staircase. J. Phys. C 16, 2497-2508 (1983)

18. Gardner, C., Miekisz, J., Radin, C., van Enter, A.C.D.: Fractal symmetry in an Ising model. J. Phys. A 22, L 1019-L 1023 (1989)

19. Keane, M.: Generalized Morse sequences. Zeit. Wahr. 10, 335-353 (1968)

20. Israel, R.B.: Existence of phase transitions for long-range interactions. Commun. Math. Phys. 43, 59-68 (1975)

21. Israel, R.B.: Convexity in the theory of lattice gases. Chap. 5, Princeton: NJ: Princeton, University Press 1979

22. Georgii, H.O.: Gibbs measures and phase transitions. Chap. 16. Berlin, New York: de Gruyter 1988

23. Bishop, E., Phelps, R.P.: The support functionals of a convex set. AMS Proceedings of Symposia in Pure Mathematics. 7, 27-35 (1963)

24. Miękisz, J.: How low temperature causes long-range order. J. Phys. A: Math. Gen. 21, L 529-L 531 (1988)

25. Miękisz, J., Radin, C.: Why solids are not really crystalline. Phys. Rev. B 39, 1950-1952 (1989)

26. Radin, C.: Ordering in lattice gases at low temperature. J. Phys. A: Math. Gen. 22, 317-319 (1989)

27. Ruelle, D.: On manifolds of phase coexistence. Theor. Math. Phys. 30, 24-29 (1977)

28. Daniëls, H.A.M., van Enter, A.C.D.: Differentiability properties of the pressure of lattice systems. Commun. Math. Phys. 71, 65-76 (1980)

29. van Enter, A.C.D.: Stability properties of phase diagrams in lattice systems. Groningen thesis (1981)

30. van Enter, A.C.D.: A note on the stability of phase diagrams in lattice systems. Commun. Math. Phys. 79, 25-32 (1981)

31. Sokal, A.D.: More surprises in the general theory of lattice systems. Commun. Math. Phys. 86, 327-336 (1982)

32. Israel, R.B.: Generic triviality of phase diagrams in spaces of long-range interactions. Commun. Math. Phys. 106, 459-466 (1986)

33. Dobrushin R.L.: Gibbs state describing the coexistence of phases for the three-dimensional Ising model. Theor. Prob. Appl. 17, 582-602 (1972) 
